\title{
Temporal Coherence of Photons Emitted by Single Nitrogen-Vacancy Defect Centers in Diamond Using Optical Rabi-Oscillations
}

\author{
A. Batalov, ${ }^{1}$ C. Zierl, ${ }^{1}$ T. Gaebel,,${ }^{1}$ P. Neumann, ${ }^{1}$ I.-Y. Chan, ${ }^{1}$ G. Balasubramanian, ${ }^{1}$ \\ P. R. Hemmer, ${ }^{2}$ F. Jelezko, ${ }^{1, *}$ and J. Wrachtrup ${ }^{1}$ \\ ${ }^{1}$ 3. Physikalisches Institut, Universität Stuttgart, 70550 Stuttgart, Germany \\ ${ }^{2}$ Department of Electrical and Computer Engineering, Texas A\&M University, College Station, USA \\ (Received 7 October 2007; revised manuscript received 16 December 2007; published 21 February 2008)

\begin{abstract}
Photon interference among distant quantum emitters is a promising method to generate large scale quantum networks. Interference is best achieved when photons show long coherence times. For the nitrogen-vacancy defect center in diamond we measure the coherence times of photons via optically induced Rabi oscillations. Experiments reveal a close to Fourier-transform (i.e., lifetime) limited width of photons emitted even when averaged over minutes. The projected contrast of two-photon interference (0.8) is high enough to envisage applications in quantum information processing. We report 12 and $7.8 \mathrm{~ns}$ excited state lifetimes depending on the spin state of the defect.
\end{abstract}

Coherent control of single quantum systems and the generation of nonclassical states has attracted widespread attention because of their application in quantum physics and quantum information science. Solid state systems are often considered to be promising and also difficult because of inhomogeneities and fast dephasing. Spins in solids, for example, associated with quantum dots or single dopant atoms offer promising figures of merit for both parameters. As a particular example, the nitrogen-vacancy (NV) defect in ultrapure diamond shows a long spin phase memory time $(0.35 \mathrm{~ms})$ [1] even at ambient conditions due to spin-free and rigid lattice. In addition to its excellent spin properties, spin selectivity of optical transitions of the NV defect allows initialization and readout of the spin state with sensitivity routinely reaching a single atom [2].

The narrow spin resonance transitions of single defects make them a sensitive magnetometer at the nanoscale. As an example, it was demonstrated that the electron spin associated with a single NV defect can be used for reading out spin states of proximal nuclear [3] and electron spins $[1,4,5]$. Magnetic coupling between electron spin of NV defects and neighboring nuclei was used as a resource for generating entangled states [6]. Such multispin entanglement is a crucial element for quantum computation and communications protocols [7]. However, the generation of entanglement using magnetic dipolar coupling is limited to closely spaced spins. The maximum distance where spinspin interaction can be used for controlling nonlocal quantum states depends on coherence time and strength of spinspin interaction. Although coherences associated with electron and nuclear spins in diamond are particularly long, a few nanometers distance is a realistic limit for magnetic coupling. One way to gain entanglement over larger distance is to use the coupling of spin state to optical transitions $[8,9]$. Such generation of entanglement over long distance via interference of photons recently attracted considerable interest [10]. Experimental demonstration of entanglement between stationary and flying qubits $[11,12]$ followed by realization of interference of photon pairs from distant trapped ions [13] and their entanglement via photonic channels [14] is an important breakthrough for modern quantum physics.

The generation of two-photon interference in solid state systems remains challenging. The major difficulties that solid state systems are facing are the coherence properties of photons and the inhomogeneous distribution of transition frequencies. By using appropriate control parameters, the inhomogeneity of a solid state system can be eliminated. For single NV defects tuning of transition frequencies has been reported previously [15]. However, dynamic inhomogeneity (variation of optical transition frequency related to different relaxation processes in solids) leads to mapping of environmental fluctuations into the frequency of photons. This makes two photons emitted by nominally identical systems at least in principle distinguishable destroying the contrast of the two-photon interference. Despite those challenges, several well-isolated solid state systems have been investigated in detail $[16,17]$. In the above mentioned context we address a single NV defect in diamond. The NV defect was proven to be an efficient source of broadband photons at ambient conditions $[18,19]$ allowing spectacular experiments of single photon interference [20]. Here we present experimental evidence of nearly transform-limited transition of single NV centers at cryogenic temperature. This is proven in two steps, first by measuring the excited state lifetime for different spin states, and subsequently generating optical Rabi oscillations. Surprisingly, our experiment reveals new insights into the photophysics of the NV defects allowing the achievement of high visibility of ESR Rabi oscillation at ambient conditions.

The structure and energy levels of the NV defect in diamond are shown in Fig. 1. The defect consists of a substitutional nitrogen atom and a vacancy in nearest 


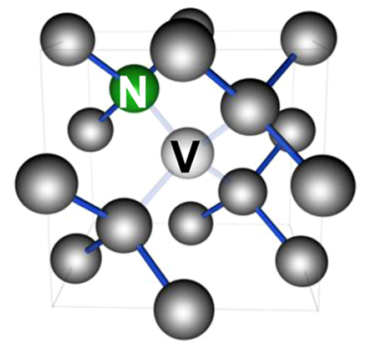

(a)

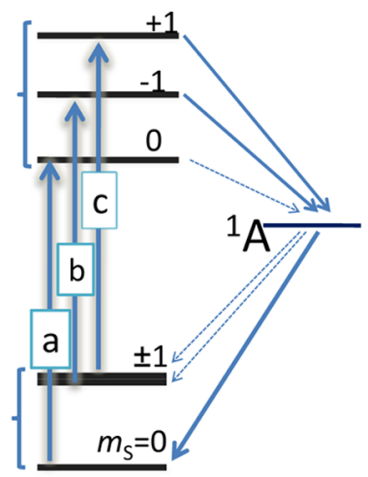

(b)
FIG. 1 (color online). (a) Structure of the NV center in diamond. The NV center comprises a substitutional nitrogen $(\mathrm{N})$, and a neighboring vacancy (V). (b) The scheme of energy levels of the NV defect center. Thick arrows indicate spin-selective excitation and fluorescence emission pathways (transitions a,b and c).

neighbor lattice position. The negatively charged NV center, which is relevant for our experiments, comprises six electrons, two of which are unpaired. The ground ${ }^{3} A$ state is a spin triplet with zero-field splitting of $2.88 \mathrm{GHz}$ between $m_{S}=0$ and $m_{S}= \pm 1$ states. The structure of the excited ${ }^{3} E$ state is governed by spin-orbit and spin-spin interactions [21]. It is well established that at least one singlet state $\left({ }^{1} A\right)$ is lying between the ground and excited triplet state [22]. Transitions between triplet and singlet states govern the spin polarization dynamics of the NV defect. It was demonstrated experimentally that the intersystem crossing (ISC) transitions ${ }^{3} E \rightarrow{ }^{1} A$ are strongly spin selective with the shelving rate from the $m_{S}=0$ sublevel being much smaller than those from $m_{S}= \pm 1$ sublevels. The rates of ISC transitions towards the groundstate triplet ${ }^{1} A \rightarrow{ }^{3} A$ are also spin selective, but here the $m_{S}=0$ state is mostly populated. Hence after a few optical excitation emission cycles a strong spin polarization of the ground-state $m_{S}=0$ spin sublevel is established.

As a first step the excited state lifetime of the NV center was measured. Efficient optical pumping leads to spin polarization into the $m_{S}=0$ sublevel as discussed above. Hence it is difficult to measure the decay of the excited state $m_{S}= \pm 1$ sublevels directly. It was suggested to use very low repetition rate lasers to allow the system to reach thermal equilibrium between optical pulses [22]. The long reported $T_{1}$ time of the spin state [23] (265 s) makes such an experiment unfeasible for single atoms. In order to detect the decay of the excited state we have chosen a different experimental approach described in Fig. 2. The $\mathrm{NV}$ center at room temperature was first polarized into the $m_{S}=0$ state by a microsecond optical pulse with $\lambda=$ $532 \mathrm{~nm}$. After that the fluorescence decay was measured using subpicosecond excitation pulses which were synchronized with single photon counting data acquisition.

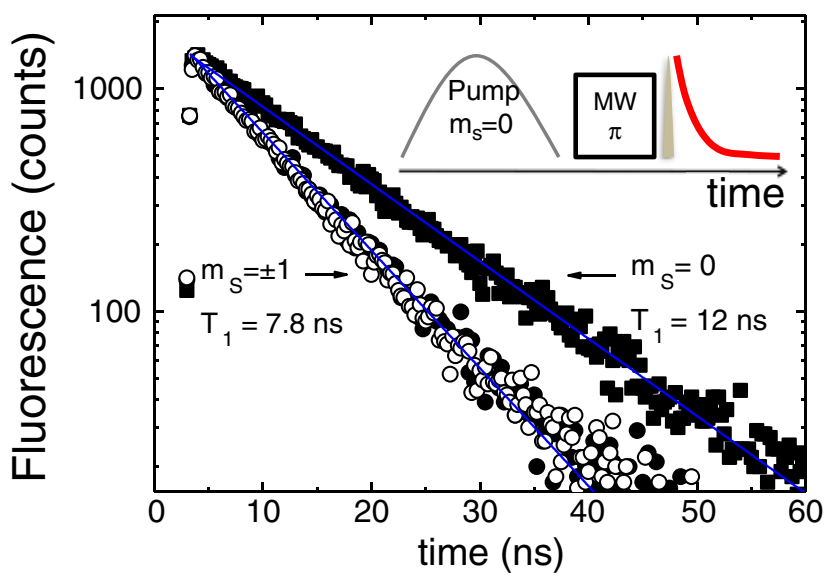

FIG. 2 (color online). Spin-selective decay curves of a single NV defect at ambient conditions. The inset shows the time sequence of the experiment. The $m_{S}=0$ spin states have been initialized by a $\mu$ s long optical pulse followed by long ( $\mu \mathrm{s})$ delay. Next, the center was excited by 150 fs laser pulse and the fluorescence decay was recorded (this sequence was used for lifetime measurements of $m_{S}=0$ state, squares, corresponds to transition a shown in Fig. 1). Introduction of a spin-selective microwave $\pi$ pulse after the polarization pulse initializes the system into $m_{S}= \pm 1$ prior to lifetime measurements (open and filled circles, transition b, c shown in Fig. 1). Solid lines are single exponential fit curves.

For measuring the decay of the $m_{S}= \pm 1$ states a microwave $\pi$ pulse was introduced after the spin polarization pulse.

Results of such measurements are shown in Fig. 2. Fluorescence decay curves follow single exponential decay with time constants of $12.0 \mathrm{~ns}$ for the $m_{S}=0$ state and $7.8 \mathrm{~ns}$ for $m_{S}= \pm 1$, respectively. The decay of the $m_{S}=0$ state found here is in good agreement with a previously reported lifetime [24], whereas its dependence on the spin quantum number is surprising at first sight. It is remarkable that the total number of photons emitted from excited $m_{S}= \pm 1$ states is smaller compared to $m_{S}=0$ state. This indicates that the difference in lifetime is due to the $m_{S}$ dependent nonradiative passage into the metastable state and not to the change of the transition oscillator strength. The total excited state decay rate is the sum of the radiative decay rate and intersystem crossing rate, $k_{\mathrm{fl} .}=k_{3 E-3 A}+k_{3 E-1 A}$. Since $k_{3 E-1 A}$ is different for $m_{s}=$ 0 and $\pm 1, k_{\mathrm{fl}}$ depends on the spin state. From our experiments a difference in the intersystem crossing rate $k_{3 E-1 A}$ among the spin states of $4.5 \times 10^{-7} \mathrm{~s}^{-1}$ can be deduced which is in excellent agreement with recent theoretical predictions [22].

The above mentioned finding allows for an improvement of single spin experiments. The decay into the metastable state defines the contrast in $\mathrm{cw}$ and pulsed optically detected ESR experiments. Under stationary conditions this contrast never exceeds $30 \%$. The significant difference in fluorescence lifetimes can be used for improving the opti- 
cal readout of the spin state. This can be made by gating the detection channel and selecting only the long lifetime photons. Figure 3 shows the result of such selection using a gating window of $10 \mathrm{~ns}$ with a $100 \mathrm{~ns}$ delay after the laser pulse. The visibility is improved by a factor of 2 when compared to the previously reported scheme [2,25]. The visibility of the ESR Rabi oscillations can be improved to an arbitrary value by making delay time longer. The price to pay is loss of the count rate leading to a longer integrating time.

At low temperatures not only the lifetime, but also the frequency of photons associated with specific transition carry information about the spin state [26]. This spinphoton frequency correspondence would potentially allow entangling of two distant NV defects using photon interference. In order to demonstrate that such remote entanglement via projective state measurement is applicable to NV diamond, the coherence time of photons should be as long as possible. It was shown that nitrogen-free samples usually show narrow spectral lines. Recently lifetimelimited excitation lines were recorded under weak laser illumination [15]. However, the question about the possibility to observe Fourier-transform-limited photons under strong optical excitation remains to be addressed.

The figure of merit for photons in the interference experiments mentioned above is the Fourier-transform relation between spectral $\Delta \nu$ and temporal $\Delta \tau$ profiles $\Delta \nu \Delta \tau=\frac{1}{2 \pi}$. Both parameters are related to the coherence $T_{2}$ and relaxation $T_{1}$ time of the corresponding optical

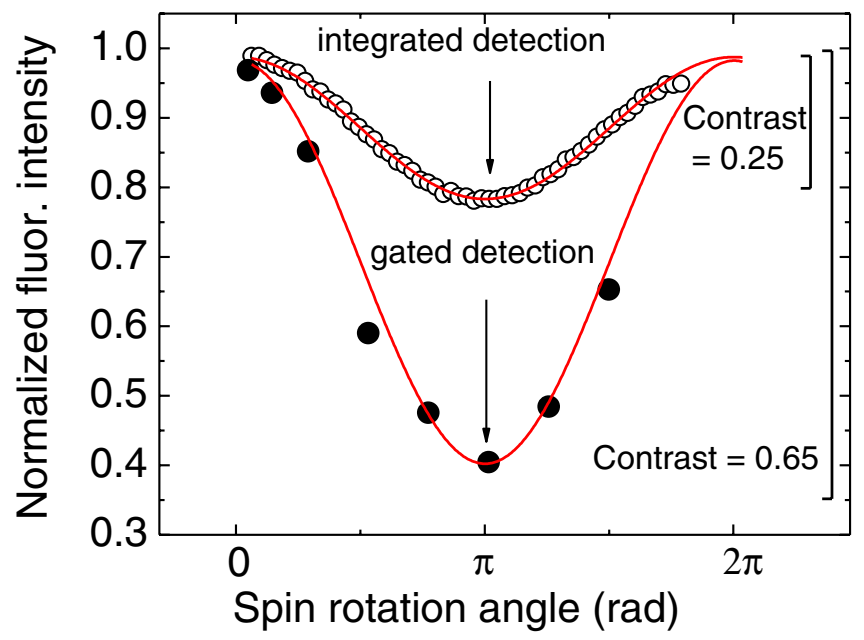

FIG. 3 (color online). Rabi oscillations of a single NV electron spin recorded using temporal gating (solid circles). After initialization of the NV center into the $m_{S}=0$ state a resonant microwave pulse of variable length was applied. Consecutively a short (100 fs) pulse was used for reading out. In order to make the detection scheme sensitive to the $m_{S}=0$ state population, only photons with long lifetime were selected by choosing the delay of detection window to be $100 \mathrm{~ns}$. Measurement based on discrimination of spin states via total fluorescence is shown for comparison (open circles). transitions via $\Delta \nu=\frac{1}{2 \pi T_{2}}$ and $\Delta \tau=2 T_{1}$. Hence, fully coherent wave packets are satisfying the criterion $\frac{2 T_{1}}{T_{2}}=1$. Several solid state systems are approaching this limit with achieved ratios of $\frac{2 T_{1}}{T_{2}}=1.6$ for single organic molecules [17] and $\frac{2 T_{1}}{T_{2}}=1.5$ for semiconductor quantum dots [27].

In order to characterize the coherence properties of the optical transitions we have used fluorescence excitation spectroscopy. The sample was a natural diamond immersed into superfluid helium $(T \sim 2 \mathrm{~K})$. A narrow single frequency dye laser (linewidth $500 \mathrm{kHz}$ ) is tuned to resonance on the electronic transition of $\mathrm{NV}$ and the second-order correlation function of the broad Stokes-shifted emission into the phonon sideband is recorded. Although these broadband photons cannot be used in quantum computation experiments directly, they carry important information about the coherence properties of transition. In general, the homogeneous linewidth can be defined as $\Gamma_{2}=\frac{1}{T_{2}}=\frac{1}{2 T_{1}}+$ $\frac{1}{T_{2}^{*}}$. Here $T_{2}^{*}$ is the pure dephasing time. Such dephasing can be considered as a source of losses in two-photon interference experiments. If dephasing is present, the depth of the Hong-Ou-Mandel dip is reduced to $\frac{T_{2}}{2 T_{1}}$ [28]. Thus, it is of crucial importance to characterize $T_{2}^{*}$ of the photon source. Since under realistic conditions a single photon source has to be driven strongly, it must be measured under saturating optical excitation. A single transition associated with the long-cycling $m_{S}=0$ state is visible in the fluorescence excitation spectrum of the defect [21]. The derivation of $T_{2}^{*}$ from the linewidth of the detected transition is complicated due to the saturation broadening, whereas the second-order intensity correlation function of the emitted photons $g^{(2)}(\tau)=\langle I(t) I(t+\tau)\rangle /\langle I(t)\rangle^{2}$ allows extracting it in a more straightforward way. The experimental measurements of $g^{(2)}(\tau)$ (Fig. 4) show the signature of optically induced Rabi oscillations with a decay defined by the radiative lifetime of the excited state and pure dephasing. Since the metastable state population occurs on a long time scale for the $m_{S}=0$ state, the system can be analyzed in terms of optical Bloch equations for a two-level system. The corresponding second-order intensity correlation function can be written as [29]

$$
\begin{aligned}
g^{(2)}(\tau)= & -\exp \left[-\left(\frac{3}{4 T_{1}}+\frac{1}{2 T_{2}^{*}}\right) \tau\right] \\
& \times\left[\left(\frac{3}{4 T_{1}}+\frac{1}{2 T_{2}^{*}}\right) \frac{1}{\Omega} \sin (\Omega \tau)+\cos (\Omega \tau)\right] .
\end{aligned}
$$

Here $\Omega$ is the optical Rabi frequency. Fit functions with $T_{2}^{*}$ as fitting parameter ( $T_{1}$ is a fixed parameter measured independently as described above) are presented together with experimental data. There is a clear indication that dephasing occurs at the hard driving laser field. Note that at $0.44 \mathrm{GHz}$ driving frequency the pure dephasing time exceeds 80 ns (lower curve), which corresponds to $77 \%$ of Mandel dip depth for a two-photon interference experi- 


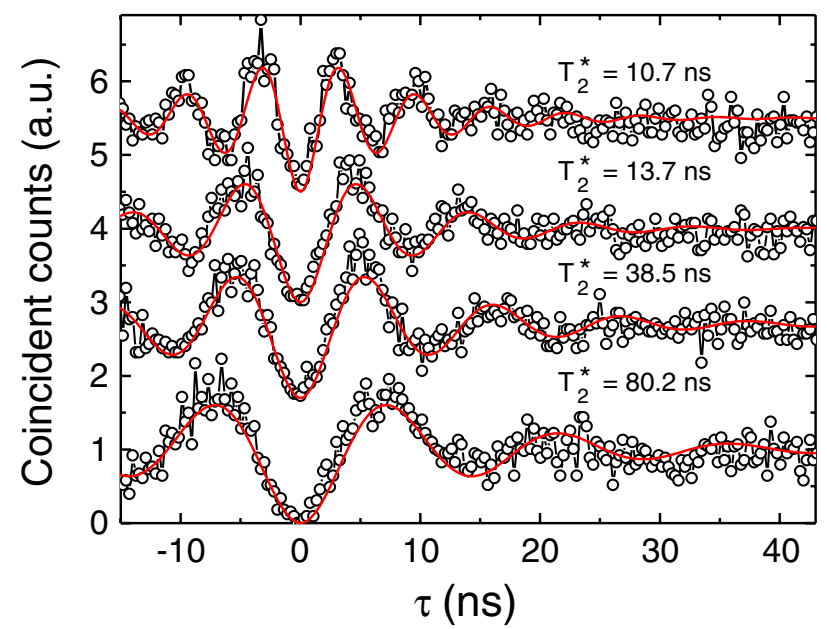

FIG. 4 (color online). Second-order fluorescence intensity autocorrelation function for the $\mathrm{NV}$ center at low temperature under resonant excitation of $m_{S}=0$ spin state (corresponds to transition a shown in Fig. 1). The Rabi frequency of the excitation laser was varied for different curves and equals to $0.44,0.59$, 0.67 , and $1.00 \mathrm{GHz}$ (increase from bottom to top). The fit functions (solid lines) are based on Eq. (1) (see text).

ment. It is important to mention that the measured $T_{2}^{*}$ is characteristic for the whole acquisition time of the experiment (a few tens of minutes). During this time approximately $10^{10}$ photons were emitted and $3 \times 10^{7}$ were detected. Previously reported two-photon interference experiments on single quantum dots rely on indistinguishability of two consecutive photons separated by a time interval of a few nanoseconds [27]. Slow spectral jumps would probably destroy coalescence contrast on a longer time scale, which is not the case for NV centers.

In summary, we show an important new insight into the coherence properties of NV centers in diamond. The NV defects show narrow linewidth of optical transition with minor contributions from pure dephasing when compared to broadening via radiative decay. To obtain a single photon stream for two-photon interference experiments, the NV center must be excited nonresonantly and the photons associated with the zero-phonon line must be selected for detection. Alternatively, fast optical switches can be used to separate the excitation pulse from the fluorescence photons when resonant excitation is used. The novel contrast mechanism for single spin ESR at ambient condition based on a temporal filtering of photons might help to facilitate the detection and spin manipulations in the quantum register.

This work supported in part by DFG (Project No. SFB/ TRR 21 and No. WR 26/16), EU (QAP, EQUIND, NEDQIT) and foundation "Landesstiftung B-W" (project "Atomoptik").

*f.jelezko@physik.uni-stuttgart.de

[1] T. Gaebel et al., Nature Phys. 2, 408 (2006).

[2] F. Jelezko et al., Phys. Rev. Lett. 92, 076401 (2004).

[3] L. Childress et al., Science 314, 281 (2006).

[4] R. J. Epstein et al., Nature Phys. 1, 94 (2005).

[5] R. Hanson et al., Phys. Rev. Lett. 97, 087601 (2006).

[6] M. V. G. Dutt et al., Science 316, 1312 (2007).

[7] P. Zoller et al., Eur. Phys. J. D 36, 203 (2005).

[8] L. Childress et al., Phys. Rev. A 72, 052330 (2005).

[9] L. Childress et al., Phys. Rev. Lett. 96, 070504 (2006).

[10] S. D. Barrett and P. Kok, Phys. Rev. A 71, 060310 (2005).

[11] B. B. Blinov et al., Nature (London) 428, 153 (2004).

[12] J. Volz et al., Phys. Rev. Lett. 96, 030404 (2006).

[13] P. Maunz et al., Nature Phys. 3, 538 (2007).

[14] D. L. Moehring et al., Nature (London) 449, 68 (2007).

[15] P. Tamarat et al., Phys. Rev. Lett. 97, 083002 (2006).

[16] C. Santori et al., Phys. Rev. Lett. 97, 247401 (2006).

[17] A. Kiraz et al., Phys. Rev. Lett. 94, 223602 (2005).

[18] R. Brouri et al., Opt. Lett. 25, 1294 (2000).

[19] C. Kurtsiefer et al., Phys. Rev. Lett. 85, 290 (2000).

[20] V. Jacques et al., Science 315, 966 (2007).

[21] P. Tamarat et al., arXiv:cond-mat/0610357.

[22] N. B. Manson and R. L. McMurtrie, J. Lumin. 127, 98 (2007).

[23] J. Harrison, M. J. Sellars, and N. B. Manson, Diam. Relat. Mater. 15, 586 (2006).

[24] A. T. Collins, M. F. Thomaz, and M. I. B. Jorge, J. Phys. C 16, 2177 (1983).

[25] F. Jelezko et al., Phys. Rev. Lett. 93, 130501 (2004).

[26] F. Jelezko et al., Appl. Phys. Lett. 81, 2160 (2002).

[27] C. Santori et al., Nature (London) 419, 594 (2002).

[28] J. Bylander, I. Robert-Philip, and I. Abram, Eur. Phys. J. D 22, 295 (2003).

[29] T. Basche et al., Phys. Rev. Lett. 69, 1516 (1992). 